\title{
Combined application of rapamycin and atorvastatin improves lipid metabolism in apolipoprotein E-deficient mice with chronic kidney disease
}

\author{
Eun Ju Song ${ }^{1,2, \#}$, Sanghyun $\mathrm{Ahn}^{3, \#}$, Seung-Kee Min ${ }^{3}$, Jongwon $\mathrm{Ha}^{3, *} \mathcal{E}$ Goo Taeg $\mathrm{Oh}^{1, *}$ \\ ${ }^{1}$ Immune and Vascular Cell Network Research Center, National Creative Initiatives, Department of Life Sciences, Ewha Womans \\ University, Seoul 03760, ${ }^{2}$ Department of Veterinary Physiology, BK21 PLUS Program for Creative Veterinary Science Research, Research \\ Institute for Veterinary Science and College of Veterinary Medicine, Seoul National University, Seoul 08826, ${ }^{3}$ Department of Surgery, Seoul \\ National University College of Medicine, Seoul 03080, Korea
}

\begin{abstract}
Atherosclerosis arising from the pro-inflammatory conditions associated with chronic kidney disease (CKD) increases major cardiovascular morbidity and mortality. Rapamycin (RAPA) is known to inhibit atherosclerosis under CKD and non-CKD conditions, but it can cause dyslipidemia; thus, the co-application of lipid-lowering agents is recommended. Atorvastatin (ATV) has been widely used to reduce serum lipids levels, but its synergistic effect with RAPA in CKD remains unclear. Here, we analyzed the effect of their combined treatment on atherosclerosis stimulated by CKD in apolipoprotein E-deficient $\left(\mathrm{ApoE}^{-/-}\right)$mice. Oil Red $O$ staining revealed that treatment with RAPA and RAPA+ ATV, but not ATV alone, significantly decreased the atherosclerotic lesions in the aorta and aortic sinus, compared to those seen in the control (CKD) group. The co-administration of RAPA and ATV improved the serum lipid profile and raised the expression levels of proteins involved in reverse cholesterol transport (LXR $\alpha$, CYP7A1, ABCG1, PPAR $\gamma$, ApoA1) in the liver. The CKD group showed increased levels of various genes encoding atherosclerosispromoting cytokines in the spleen (Tnf- $\alpha, I I-6$ and II-1 $\beta$ ) and aorta (Tnf- $\alpha$ and II-4), and these increases were attenuated by RAPA treatment. ATV and RAPA+ATV decreased the levels of Tnf- $\alpha$ and $I I-1 \beta$ in the spleen, but not in the aorta. Together, these results indicate that, in CKD-induced $\mathrm{ApoE}^{-1-}$ mice, RAPA significantly reduces the development of atherosclerosis by regulating the expression of inflammatory cytokines and the co-application of ATV improves lipid metabolism. [BMB Reports 2021; 54(3): 170-175]
\end{abstract}

*Corresponding author. Goo Taeg Oh, Tel: +82-2-3277-4128; Fax: +82-2-3277-3760; E-mail: gootaeg@ewha.ac.kr; Jongwon Ha, Tel: +82-2-2072-2991; Fax: +82-2-766-3971; E-mail: jwhamd@snu.ac.kr ${ }^{\#}$ These authors contributed equally to this work.

https://doi.org/10.5483/BMBRep.2021.54.3.136

Received 23 June 2020, Revised 27 July 2020 ,

Accepted 10 September 2020

Keywords: Atherosclerosis, Atorvastatin, Chronic kidney disease, Co-administration, Rapamycin

\section{INTRODUCTION}

Atherosclerosis and arterial calcification are more frequent and severe in patients with chronic kidney disease (CKD) than in the general population (1). Uremia in CKD patients precipitates oxidative stress and inflammation in the arteries and stimulates plaque formation, in a process that is called accelerated atherosclerosis $(2,3)$. As a result, CKD patients exhibit increased morbidity and mortality due to cardiovascular diseases (4).

The pathogenic mechanism of atherosclerosis in CKD can be explained by an imbalance of electrolytes, such as calcium (Ca) and phosphate $(\mathrm{P})$, and a loss of vascular smooth muscle cell (VSMC) function (5). Because the kidney is the main organ for cytokine removal, CKD patients exhibit cytokine dysregulation and persistent inflammation, which can stimulate vascular cell senescence (6-8). CKD also promotes dyslipidemia; this is an alteration of cholesterol homeostasis that includes increased low-density lipoprotein cholesterol (LDL-C) and decreased high-density lipoprotein dholesterol (HDL-C) levels, and is known to exacerbate atherosclerosis (9).

Several studies have reported that rapamycin (RAPA) suppresses the development of atherosclerosis and arterial calcification (10, 11). However, RAPA also has been associated with hyperlipidemia in renal transplant recipients and CKD patients, because mTOR inhibition reduces the plasma lipid clearance by inhibiting the activity of lipases required for the catabolism of circulating lipoproteins and altering the expression of enzymes for fatty acid uptake and storage $(12,13)$. Thus, it is recommended that lipid-lowering agents be administered together (14). The statin, atorvastatin (ATV), has been widely used to prevent major cardiovascular complications associated with hypercholesterolemia and dyslipidemia. ATV reportedly plays an anti-inflammatory role by inhibiting the production of tumor necrosis factor (TNF)- $\alpha$ and protecting VSMCs against TGF- $\beta 1$-mediated stimulation, and thereby protects against atherogenesis $(15,16)$. Although ATV shows a protective effect against cardiovascular disease, its use in CKD patients has been limited to date (17). A previous study found that ATV significantly reduced total cholesterol levels and LDL-C independent of CKD, and decreased triglyceride levels more in patients with CKD than in 
those without CKD (18). However, the potential synergism of RAPA and ATV (RAPA + ATV) co-treatment has not been studied in terms of atherosclerosis inhibition, especially in CKD.

To understand the mechanism of CKD-accelerated atherosclerosis in depth, in vivo CKD models are required. Although several mouse models have been developed for investigating the mechanisms of atherosclerosis caused by CKD, limitations exist because the genetic manipulations or inducing methods are associated with various degrees of renal failure (19). In this study, we established a distinct CKD mouse model promoting atherosclerosis compared with sham operated mice. And we investigated the effects of RAPA + ATV on the regulation of inflammatory cytokines and dyslipidemia to prevent the development of atherosclerosis in CKD-induced apolipoprotein E-deficient $\left(\mathrm{ApoE}^{-/-}\right)$mice.

\section{RESULTS}

\section{RAPA ameliorates CKD-associated atherosclerosis}

To establish a consistent CKD mouse model, we used a twostep surgical nephrectomy in 8-week-old female $A p o E^{-l-}$ mice (Supplemental Fig. 1A). We first evaluated the serum chemistry (Table 1), and found that the levels of blood urea nitrogen (BUN), creatinine, and calcium were markedly increased in the sera of mice subjected to surgical nephrectomy compared to sera in sham-operated mice. However, RAPA and/or ATV did not decrease the uremia and the hypercalcemia associated with CKD in mice. No significant difference between groups was seen in the serum phosphate level.

To find the effect of RAPA and/or ATV on atherosclerotic lesions accelerated by CKD, we established the CKD model as described above, and the mice were further fed a Western diet to induce atherosclerosis. This model was used in all subsequent experiments. Oil red $\mathrm{O}$ staining showed that the CKD group exhibited more atherosclerotic plaque formation in the whole aorta than did the Sham group (Fig. 1A). The RAPA and RAPA+ ATV groups exhibited significant reductions of the atherosclerotic lesions, whereas the ATV group did not differ from the CKD group. There was no significant difference between the RAPA and RAPA + ATV groups in this parameter. We observed similar results when we analyzed the stained aortic sinuses of mouse hearts (Fig. 1B). Together, these findings indicate that RAPA reduces the atherosclerosis associated with CKD in this model, but ATV has no additional effect on this parameter, alone or in combination with RAPA.

\section{Combination of RAPA and ATV improves lipid metabolism in CKD mice}

It has been reported that CKD patients may exhibit dyslipidemia, which is a major risk factor for the development of cardiovascular
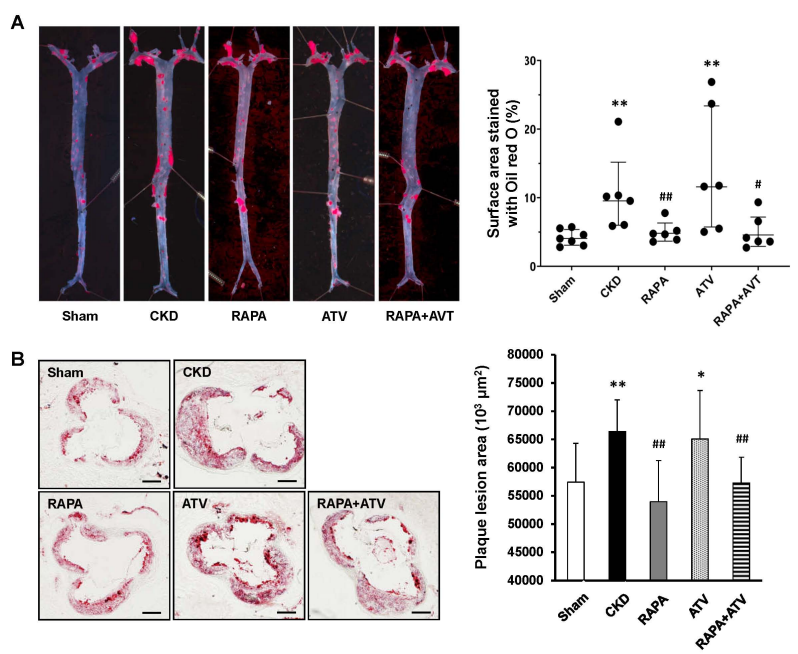

Fig. 1. RAPA ameliorates the formation of atherosclerotic plaques, as assessed by Oil red $\mathrm{O}$ staining. (A) Representative en face images of whole aortas (left) and quantification of lesion areas from the indicated groups (right) ( $A p o E^{-1-} ; \mathrm{n}=6-7$ per group). (B) Representative images of frozen sections of aortic sinuses (left) and quantification of plaque areas on aortic sinuses of the indicated groups (right) ( $\mathrm{n}=3-5$ per group). Data are shown as mean + SEM $* \mathrm{P}<0.05, * * \mathrm{P}<0.01$ compared with the Sham group; ${ }^{\#} \mathrm{P}<0.05,{ }^{\#} \mathrm{P}<0.01$ compared with the CKD group.

Table 1. Body weight and laboratory data of $A p o E^{-1-}$ mice with CKD at the end of the study

\begin{tabular}{|c|c|c|c|c|c|}
\hline & Sham & CKD & RAPA & ATV & RAPA + ATV \\
\hline Body weight (g) & $22.9 \pm 1.2$ & $* 20.4 \pm 2.4$ & $* 20.3 \pm 3.0$ & $* 20.1 \pm 4.1$ & ${ }^{\#} 22.7 \pm 1.5$ \\
\hline BUN, mg/dl & $18.7 \pm 3.9$ & $* 92.8 \pm 14.4$ & $* 78.9 \pm 32.8$ & $* 79.5 \pm 19.5$ & $* 65.8 \pm 25.2$ \\
\hline Creatinine, mg/dl & $0.28 \pm 0.02$ & $* 0.47 \pm 0.03$ & $* 0.65 \pm 0.13$ & $* 0.59 \pm 0.19$ & $* 0.56 \pm 0.14$ \\
\hline $\mathrm{B} / \mathrm{C}$ ratio & $44.4 \pm 39.8$ & $* 195.3 \pm 19.2$ & $* 111.8 \pm 28.9$ & $* 128.8 \pm 24.3$ & $* 151.3 \pm 23.3$ \\
\hline Calcium, mg/dl & $9.78 \pm 0.39$ & $* 11.27 \pm 1.48$ & $* 10.10 \pm 0.67$ & $* 10.17 \pm 0.7$ & $* 11.08 \pm 0.42$ \\
\hline Phosphate, mg/dl & $7.23 \pm 0.79$ & $8.0 \pm 2.0$ & $6.57 \pm 1.78$ & $6.73 \pm 1.08$ & $8.72 \pm 1$ \\
\hline Total protein, g/dl & $6.13 \pm 1.4$ & $8.27 \pm 3.33$ & $7.94 \pm 2.86$ & $4.45 \pm 0.31$ & $9.21 \pm 4.76$ \\
\hline Albumin, g/dl & $1.67 \pm 0.21$ & $1.77 \pm 0.4$ & $1.81 \pm 0.39$ & $1.28 \pm 0.05$ & $1.99 \pm 0.62$ \\
\hline
\end{tabular}

BUN: blood urea nitrogen; B/C: BUN/Creatinine; AST: aspartate aminotransferase; ALT: alanine aminotransferase; ALP: alkaline phosphatase. Data are shown as mean $\pm \mathrm{SEM},{ }^{*} \mathrm{P}<0.05,{ }^{* * P}<0.01$ compared with Sham group, ${ }^{\#} \mathrm{P}<0.05$, ${ }^{\# \#} \mathrm{P}<0.01$ compared with CKD group. 
disease (20). To examine the serum lipid levels in our experimental system, we fasted animals for 4 hours, sacrificed them, and collected whole blood for serum chemistry. The serum levels of total cholesterol and triglyceride were not different between the groups (Fig. 2A, B). Circulating LDL-C was significantly more increased in the CKD group than in the Sham and was inhibited in the ATV group. However, neither RAPA nor RAPA + ATV affected the LDL-C level elevated by CKD in those groups. The serum level of HDL-C in the CKD and RAPA group was similar to that in the Sham group, but the level was markedly elevated in the ATV and RAPA + ATV groups (Fig. 2C, D). Surprisingly, the HDL-C levels in the co-administration of RAPA and ATV were higher than that seen in the ATV group, with an increase that was twice those seen in the Sham and CKD groups. To explore the effect of RAPA and ATV on lipid metabolism, we used qRT-PCR to evaluate the mRNA expression levels of genes related to cholesterol metabolism, including Hmgcr, Lxro, Abcg5, Cyp7a1, Ppary, and Apoa1, in the livers of our experimental and control mice (Supplemental Fig. 2A-F). As expected, CKD increased the mRNA expression level of $\mathrm{HmgCr}$, and this change was inhibited by treatment with RAPA and/or ATV. The Hmgcr expression level did not differ between the RAPA, ATV, and RAPA + ATV groups, and the levels of all three groups were lower than that of the Sham group. CKD
A

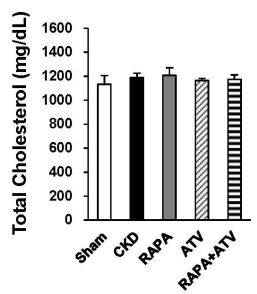

E

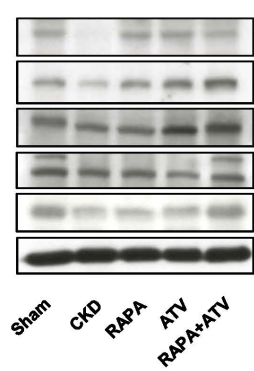

B

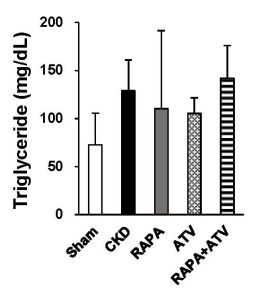

C
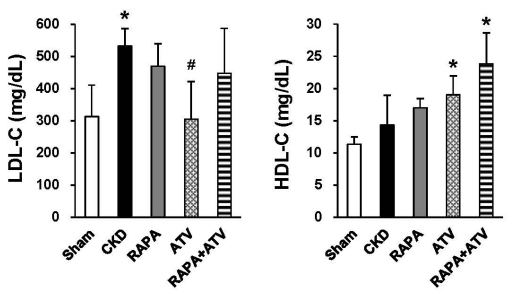

$\mathbf{F}$
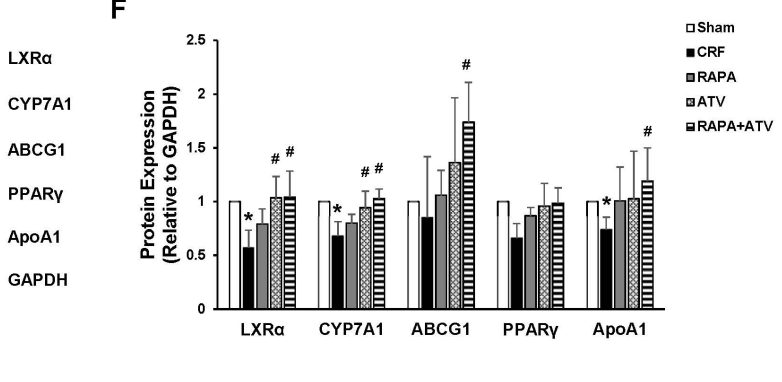

Fig. 2. Combined treatment with RAPA plus ATV has beneficial effects on lipid metabolism. Serum lipid profiles for (A) total cholesterol, (B) triglycerides, (C) lowensity lipoprotein cholesterol (LDL-C), and (D) high-density lipoprotein cholesterol (HDL-C) from the indicated groups $(\mathrm{n}=6-9)$. $(\mathrm{E}, \mathrm{F})$ The expression levels of reverse cholesterol transport-related proteins in the livers of the indicated groups ( $\mathrm{n}=5$ per group). Data are shown as mean + SEM, $* P<0.05, * * P<$ 0.01 compared with the Sham group; ${ }^{\#} \mathrm{P}<0.05,{ }^{\# \#} \mathrm{P}<0.01$ compared with the CKD group.
A

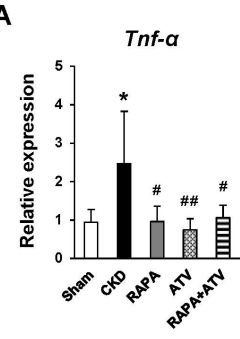

E

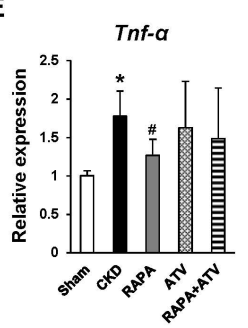

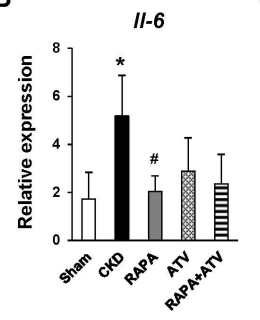

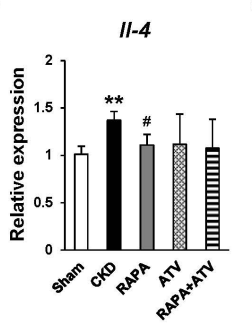

C
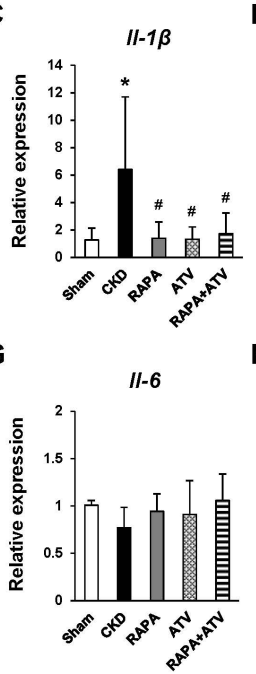

D

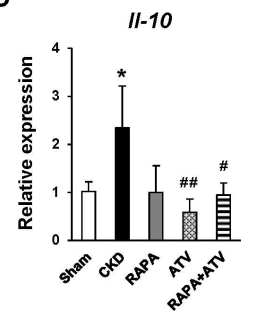

$\mathrm{H}$

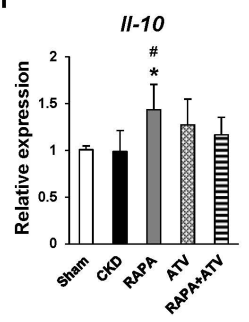

Fig. 3. The effects of RAPA and ATV on the CKD-related stimulation of proatherosclerosis cytokines in $A p o E^{-1-}$ mice. Gene expression levels of inflammatory cytokines in the spleen (A-D) and aorta $(\mathrm{E}-\mathrm{H}) \quad(\mathrm{n}=5-7$ per group). Data are shown as mean \pm SEM, $* P<0.05$, $* * \mathrm{P}<0.01$ compared with the Sham group; ${ }^{\#} \mathrm{P}<0.05,{ }^{\# \#} \mathrm{P}<0.01$ compared with the CKD group. 
tended to decrease the gene expression levels of $L x r \alpha$ compared to those in the Sham group. The gene expression of $L x r \alpha$ was significantly higher in the RAPA and/or ATV groups than in the CKD and Sham groups. The classical pathway of bile acid initiated by Cyp7a1 was more inhibited in the CKD group than in the Sham group. The RAPA + ATV group had significantly higher Cyp7a1 expression than did the CKD group. In addition, combined administration of RAPA and ATV significantly increased the mRNA level of Abcg5, Ppar $\gamma$ and Apoa 1 whereas this parameter did not differ between the RAPA, ATV, Sham, and CKD groups. We subsequently examined the expression level of proteins related to reverse cholesterol transport, including LXR $\alpha$, CYP7A1, ABCG1, PPAR $\gamma$, and ApoA1, in the mouse liver (Fig. 2E, F). The CKD group significantly reduced the expression of LXR $\alpha$, CYP7A1 and ApoA1. The decreased level of LXR $\alpha$ and CYP7A1 were recovered in similar with the Sham group by ATV administration, but the levels were not affected by RAPA treatment. Interestingly, the RAPA + ATV group had significantly elevated expression level of $L X R \alpha, C Y P 7 A 1, A B C G 1$ and ApoA1 in the liver. The expression level of PPAR $\gamma$ was slightly lowered in the CKD group, but there was no significant difference between any of the groups. Given the results, we found that co-administration of RAPA and ATV is more effective in stimulating reverse cholesterol transport and bile secretion than is ATV treatment alone. These data suggest that combining RAPA with ATV may help to mitigate dyslipidemia in CKD.

\section{$A p o E^{-1-}$ mice with CKD exhibit up-regulation of pro-inflammatory cytokine genes in the spleen and aorta, and these levels are reduced by RAPA}

Next, we used qRT-PCR to investigate the effects of RAPA and/ or ATV on the expression levels of atherogenesis-related inflammatory cytokines. In the spleen, the mRNA expression of inflammatory cytokines is known to promote atherosclerosis, including Tnf- $\alpha, I I-6$, and II-1 $\beta$, which were specifically more increased in the CKD group than in the Sham group (Fig. 3A-C). The CKD-related up-regulations of Tnf- $\alpha$ and $I I-1 \beta$ were suppressed in the ATV, RAPA, and RAPA + ATV groups, and their levels did not significantly differ between these groups. The administration of RAPA significantly inhibited the CKD-related increase of II-6 expression, whereas this level was similar among the ATV, ATV + RAPA, and CKD groups. Interestingly, II-10, an anti-inflammatory cytokine, was more increased in the CKD group than in the Sham group, and this increase was suppressed in the ATV and RAPA + ATV groups (Fig. 3D).

In the aorta, the mRNA levels of Tnf- $\alpha$ and II-4 were higher in the CKD group than in the Sham group (Fig. 3E, 3F), whereas the RAPA group had significantly lower levels of Tnf- $\alpha$ and II-4 than did the CKD group. However, ATV and RAPA + ATV did not decrease their levels like RAPA. Surprisingly, the mRNA expression level of II-6 did not differ in any of the groups (Fig. $3 G)$. The mRNA level of $I I-10$ was significantly increased in the RAPA group over that in the Sham and CKD groups (Fig.
$3 \mathrm{H})$, but this significant increase was not seen in the RAPA + ATV group. These results suggest that the administration of RAPA helps decrease the levels of atherosclerosis-promoting cytokines and increases those of atherosclerosis-suppressing cytokines, but that combined treatment with RAPA and ATV does not appear to show synergistic effects.

\section{DISCUSSION}

We herein provide the first report on how combined treatment with RAPA and ATV affects the atherogenesis stimulated by CKD. In our study, the oral administration of RAPA in CKD-induced $A p o E^{-/-}$mice ameliorated atherosclerotic lesions and inhibited the mRNA expression levels of pro-inflammatory cytokines in the spleen and aorta. These data confirm that RAPA plays an atheroprotective role by reducing the pro-inflammatory burden in CKD. Although ATV did not additionally reduce the aortic lesions, the combined use of ATV markedly increased the serum levels of $\mathrm{HDL}-\mathrm{C}$ in $A p o E^{-1-}$ mice with CDK. Thus, our results suggest that the combination therapy of RAPA plus ATV helps to alleviate the atheroprone environment of CKD.

The formation of atherosclerotic lesions can differ with the stage of renal failure. An electronic cautery-based renal injury mouse model has been used in various CKD studies because such mice exhibit substantial increases in their serum levels of BUN and creatinine $(21,22)$. However, these methods did not show sufficient or consistent renal impairment in our preliminary experiments (data not shown). We instead used high-temperature battery-operated cautery to forcefully damage the kidney and found that this procedure yielded a consistent CKD model. The serum calcium and phosphate levels of operated mice were significantly elevated, supporting the idea that our model is suitable for the mechanistic study of CKD. Since uncontrolled lipid homeostasis promotes atherosclerosis, the ability of the reverse cholesterol transport pathway to eliminate excessive serum lipids is an important factor in reducing the risk of cardiovascular disease (23). Previous study revealed that experimental chronic uremia increased serum LCL-C and stimulated atherosclerosis in mice (24). Likewise, we found more LDL-C in the serum of CKD-induced mice than in the Sham. However, there were no differences in the serum total cholesterol and triglyceride between the two groups. These findings suggest that maintaining the balance of LCL-C and HDL-C is important for improving atherosclerosis in CKD.

RAPA has been shown to significantly suppress atherosclerosis in studies using animal models with normal renal function (10, 11), but there are still concerns about adverse effects that may be associated with RAPA treatment, such as dyslipidemia. In our results, atherosclerotic lesions were substantially reduced by RAPA administration in CKD mice, but the serum lipids levels were not influenced. Several studies have reported that mTOR inhibition limits the serum lipid elimination by suppressing lipase activity and cholesterol trafficking $(12,13,25,26)$. It was also reported that RAPA treatment increases circulating PCSK9 levels, 
which are related to the increased serum LDL-C and hypercholesterolemia in patients with nephrotic syndrome $(27,28)$. These findings support the limitation of RAPA use in CKD patients. Further study will be needed to elucidate the role of RAPA in PSCK9-associated dyslipidemia. Several researchers suggested that combined treatment with a statin would reduce the possibility of RAPA-induced dyslipidemia, resulting in additional amelioration of atherosclerosis $(29,30)$. This prompted us to examine the potential of ATV to counter this disadvantage of RAPA treatment. Consistent with previous findings, our results showed that the decreased expression of LXR $\alpha$ and CYP7A1 the in the liver of CKD mice was compensated by ATV administration. LXR $\alpha$ has long been suggested as a therapeutic target against atherosclerosis, since it is implicated in cholesterol efflux and hepatic bile acid synthesis by regulating the expression of target genes associated with reverse cholesterol transport, including CYP7A1, ABCG1/5/8 and apolipoproteins $(31,32)$. Meanwhile, LXR $\alpha$ increases fatty acid (FA) and triglyceride (TG) synthesis by upregulating of genes, including SREBPC, FA synthase, and acetyl coenzyme A carboxylase (33). However, accumulated work has clearly shown the beneficial effect of $\mathrm{LXR} \alpha$ agonist against atherosclerosis. Furthermore, our study demonstrated that CKD mice treated with RAPA plus ATV gained weight (Supplemental Fig. 1B) and exhibited stabilization of the HDL-C level (Fig. 2C). The combined use of RAPA and ATV further promoted the expression of ABCG1 and ApoA1, which contribute more to cholesterol efflux than dose RAPA or ATV alone. Given that hepatic ApoA1 synthesis decreases and HDL-C level falls are common effects of renal failure (34), our study suggests that the activation of LXRo/ApoA1/ABCG1 by RAPA and ATV co-administration improves the stability of HDL-C and atherosclerosis in $\operatorname{CKD}(35,36)$. These results suggest that the combined treatment could be synergistic in eliminating the excessive serum lipids promoting atherosclerosis, and improving general conditions and long-term mortality in mice with renal failure.

Dysregulation of cytokines in CKD is associated with a significant decrease in cytokine secretion, given that the kidney is a main organ for eliminating cytokines (9). RAPA was previously shown to target pro-inflammatory cytokines and mTOR activation induced by chronic consumption of a high-fat diet (37). Indeed, we found that RAPA, ATV, and RAPA + ATV all markedly decreased the gene expression levels of atherosclerosis-promoting inflammatory cytokines such as Tnf- $\alpha, \|-6$ and $I I-1 \beta$ in the spleen. Interestingly, the mRNA level of the anti-inflammatory cytokine, II-10, was increased in the spleen tissues of the CKD group. This appears to be a compensatory mechanism intended to correct inflammation in CKD (38). In the aorta, RAPA specifically decreased the level of II-4 and increased the level of II-10, indicating that it exerts anti-inflammatory effects to prevent atherogenesis. Conversely, these levels were similar in the ATV, RAPA + ATV, and CKD groups. Several reports have suggested that ATV can have inhibitory effects on atherosclerosis in non-CKD models $(39,40)$. Thus, the previous and present results demonstrate that RAPA and ATV can both regulate the systemic inflammation environ- ment in CKD patients, but that these effects are not synergistic. In conclusion, we herein show that RAPA can play a critical role in reducing the development of atherosclerosis in the aortas of CKD-induced $A p o E^{-l-}$ mice by alleviating systemic inflammation. Although the co-administration of ATV did not further reduce atherosclerosis, it improved the lipid profile and bile acid metabolism in these mice. Thus, the combined administration of RAPA and ATV can provide synergistic effects in alleviating the cardiovascular risk associated with CKD, beyond the effects of either agent alone.

\section{MATERIALS AND METHODS}

The detailed methods are described in the "Supplementary Information".

\section{ACKNOWLEDGEMENTS}

This study was supported by the Research Resettlement Fund for the new faculty of Seoul National University, a grant from the SNUH Research Fund (04-2016-0360), and a grant from the National Research Foundation of Korea (NRF) funded by the Korean government (NRF-2012R1A3A2026454). The funders and company had no role in study design, data collection, analysis, the decision to publish, or preparation of the manuscript.

\section{CONFLICTS OF INTEREST}

The authors have no conflicting interests.

\section{REFERENCES}

1. London GM and Drueke TB (1997) Atherosclerosis and arteriosclerosis in chronic renal failure. Kidney Int 51, 1678-1695

2. Zoccali C and London G (2015) Con: vascular calcification is a surrogate marker, but not the cause of ongoing vascular disease, and it is not a treatment target in chronic kidney disease. Nephrol Dial Transplant 30, 352-357

3. Himmelfarb J, Stenvinkel P, Ikizler TA and Hakim RM (2002) The elephant in uremia: oxidant stress as a unifying concept of cardiovascular disease in uremia. Kidney Int 62, 1524-1538

4. Ketteler M, Schlieper G and Floege J (2006) Calcification and cardiovascular health: new insights into an old phenomenon. Hypertension 47, 1027-1034

5. Finch JL, Lee DH, Liapis $\mathrm{H}$ et al (2013) Phosphate restriction significantly reduces mortality in uremic rats with established vascular calcification. Kidney Int 84, 1145-1153

6. Carrero JJ, Yilmaz MI, Lindholm B and Stenvinkel P (2008) Cytokine dysregulation in chronic kidney disease: how can we treat it? Blood Purif 26, 291-299

7. Tbahriti HF, Meknassi D, Moussaoui R et al (2013) Inflammatory status in chronic renal failure: The role of homocysteinemia and pro-inflammatory cytokines. World J Nephrol 2, 31-37

8. Gisterå A and Hansson GK (2017) The immunology of atherosclerosis. Nat Rev Nephrol 13, 368-380 
9. Zewinger S, Kleber ME, Rohrer L et al (2017) Symmetric dimethylarginine, high-density lipoproteins and cardiovascular disease. Eur Heart J 38, 1597-1607

10. Kurdi A, De Meyer GR and Martinet W (2016) Potential therapeutic effects of mTOR inhibition in atherosclerosis. Br J Clin Pharmacol 82, 1267-1279

11. Ai $D$, Jiang $H$, Westerterp $M$ et al (2014) Disruption of mammalian target of rapamycin complex 1 in macrophages decreases chemokine gene expression and atherosclerosis. Circ Res $114,1576-1584$

12. Hoogeveen RC, Ballantyne CM, Pownall HJ et al (2001) Effect of sirolimus on the metabolism of ApoB100-containing lipoproteins in renal transplant patients. Transplantation 72 , $1244-1250$

13. Pallet $N$ and Legendre $C$ (2012) Adverse events associated with mTOR inhibitors. Expert Opin Drug Saf 12, 177-186

14. Kurdi A, Martinet W and De Meyer GRY (2018) mTOR Inhibition and Cardiovascular Diseases: Dyslipidemia and Atherosclerosis. Transplantation 102, S44-S46

15. Zhang X, Tian F, Kawai $\mathrm{H}$ et al (2012) Anti-inflammatory effect of amlodipine plus atorvastatin treatment on carotid atherosclerosis in zucker metabolic syndrome rats. Transl Stroke Res 3, 435-441

16. Liu D, Cui W, Liu B et al (2014) Atorvastatin protects vascular smooth muscle cells from TGF- $\beta 1$-stimulated calcification by inducing autophagy via suppression of the $\beta$-catenin pathway. Cell Physiol Biochem 33, 129-141

17. Koren MJ, Davidson MH, Wilson DJ, Fayyad RS, Zuckerman A and Reed DP (2009) Focused atorvastatin therapy in managed-care patients with coronary heart disease and CKD. Am J Kidney Dis 53, 741-750

18. Manni G, Gargaro M, Turco A et al (2018) Statins regulates inflammatory macrophage phenotype through the activation of AhR. J Immunol 200 (1 Supplement), 167.19

19. Yang HC, Zuo Y and Fogo AB (2010) Models of chronic kidney disease. Drug Discov Today Dis Models 7, 13-19

20. Mikolasevic I, Žutelija M, Mavrinac V and Orlic L (2017) Dyslipidemia in patients with chronic kidney disease: etiology and management. Int J Nephrol Renovasc Dis 10, 35-45

21. Massy ZA, Ivanovski O, Nguyen-Khoa T et al (2005) Uremia accelerates both atherosclerosis and arterial calcification in apolipoprotein E knockout mice. J Am Soc Nephrol 16, 109-116

22. Gagnon RF and Gallimore B (1988) Characterization of a mouse model of chronic uremia. Urol Res 16, 119-126

23. Annema W and Tietge UJ (2012) Regulation of reverse cholesterol transport - a comprehensive appraisal of available animal studies. Nutr Metab (Lond) 9, 25

24. Apostolov EO, Ray D, Savenka AV, Shah SV and Basnakian AG (2010) Chronic uremia stimulates LDL cabamylation and atherosclerosis. J Am Soc Nephrol 21, 1852-1857

25. Houd VP, Brûlé S, Festuccia WT et al (2010) Chronic rapamycin treatment causes glucose intolerance and hyperlipidemia by upregulating hepatic gluconeogenesis and imparing lipid deposition in adipose tissue. Diabetes 59, 1338-1348

26. Eid W, Dauner K, Courtney KC et al (2017) mTORC1 activates SREBP-2 by suppressing cholesterol trafficking to lysosome in mammalian cells. Proc Natl Acad Sci U S A 114, 7999-8004

27. Simha V, Qin S, Shah P et al (2017) Sirolimus therapy is associated with elevation in circulating PCSK9 levels in cardiac transplant patients. J Cardiovasc Trans Res 10, 9-15

28. Haas ME, Levenson AE, Sun X et al (2016) The role of proprotein convertase subtilisin/kexin type 9 in nephrotic syndromeassociated hypercholesterolemia. Circulation 134, 61-72

29. Peng N, Meng N, Wang S et al (2014) An activator of mTOR inhibits oxLDL-induced autophagy and apoptosis in vascular endothelial cells and restricts atherosclerosis in apolipoprotein $\mathrm{E}^{-1-}$ mice. Sci Rep 4, 5519

30. Martinet W, De Loof H and De Meyer GRY (2014) mTOR inhibition: a promising strategy for stabilization of atherosclerotic plaques. Atherosclerosis 233, 601-607

31. Gupta S, Pandak WM and Hylemon PB (2002) LXR $\alpha$ is the dominant regulator of CYP7A1 transcription. Biochem Biophys Res Commun 293, 338-343

32. Calkin AC and Tontonoz P (2010) Liver X recptor signaling pathways and atherosclerosis. Arterioscler Thromb Vasc Biol 30, 1513-1518

33. Chisholm JW, Hong J, Mills SA and Lawn RM (2003) The LXR ligand T0901317 induces severe lipogenesis in the $\mathrm{db} / \mathrm{db}$ diabetic mouse. J Lipid Res 44, 2039-2048

34. Schiffrin EL, Lipman ML and Mann JF (2007) Chronic Kidney Disease. Circulation 116, 85-97

35. Kennedy MA, Barrera GC, Nakamura K et al (2005) ABCG1 has a critical role in mediating cholesterol efflux to HDL and preventing cellular lipid accumulation. Cell Metabolism $1,121-131$

36. Park J, Jeong S, Yu J, Kim G, Jeong LS and Oh GT (2018) LJ-1888, a selective antagonist for the $\mathrm{A}_{3}$ adenosine receptor, ameliorates the development of atherosclerosis and hypercholesterolemia in apolipoprotein E knock-out mice. BMB Rep 51, 520-525

37. Soltani A, Bahreyni A, Boroumand $N$ et al (2018) Therapeutic potency of mTOR signaling pharmacological inhibitors in the treatment of proinflammatory diseases, current status, and perspectives. J Cell Physiol 233, 4783-4790

38. Stenvinkel P, Ketteler M, Johnson RJ et al (2005) IL-10, IL-6, and TNF-alpha: central factors in the altered cytokine network of uremia-the good, the bad, and the ugly. Kidney Int 67, 1216-1233

39. Zhao X, Liu Y, Zhong Y et al (2015) Atorvastatin Improves Inflammatory Response in Atherosclerosis by Upregulating the Expression of GARP. Mediators Inflamm 2015, 841472

40. Li H, Tao HR, Hu T et al (2010) Atorvastatin reduces calcification in rat arteries and vascular smooth muscle cells. Basic Clin Pharmacol Toxicol 107, 798-802 